\title{
Le leadership pendant et après la crise de la COVID-19
}

Les opinions exprimées dans cet éditorial sont celles de l'auteur et ne représentent pas nécessairement celles de I'Association médicale canadienne ou ses filiales.

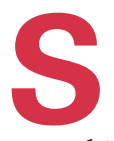

ur Internet, on trouve 1429 définitions de « leadership $\gg$. Pourtant, si on nous le demandait, nous pourrions probablement tous donner rapidement une définition que nous jurerions exacte. Cette contradiction a été mise en évidence par l'explosion relativement récente, au cours des 15 dernières années environ, du nombre de cours, de documents, de conférences et de ressources en ligne sur le concept de leadership. À défaut de clarifier en quoi consiste le leadership, ce contenu nous permet de mieux saisir les caractéristiques d'un bon leader et, plus précisément, comment celui-ci arrive à faire qu'un groupe transcende les parties qui le composent. En raison de la pandémie actuelle de maladie à coronavirus 2019 (COVID-19), qui est venue bouleverser les pratiques, il devient plus nécessaire que jamais de voir émerger un leadership exceptionnel dans le milieu chirurgical.

La plupart des concepts qui signalent un leadership fort proviennent du sport, du commerce ou des forces militaires d'élite. Ces philosophies ont servi à bâtir des équipes gagnantes dans toutes les sphères de la vie ${ }^{1}$. Nombre de chirurgiens ont déjà pratiqué des sports d'équipe de haut niveau, et ont donc une certaine expérience des principes de leadership. Dans le contexte actuel, il est quand même pertinent de les rappeler : 1) recruter des personnes plus intelligentes que soi; 2) écouter les idées de ses subalternes; 3) régler les questions importantes directement avec son équipe; 4) créer un milieu accueillant, où le personnel peut faire preuve d'honnêteté et d'authenticité et se sent à l'aise de parler de ses faiblesses (pour voir à ce que les conflits ne débordent pas); 5) établir et cultiver une mission propre à chacun (le grand objectif qui chapeaute les tâches quotidiennes), de même qu'une vision ou une mission claire, renouvelable et sans cesse actualisée pour l'ensemble de l'équipe; 6) bâtir un environnement qui favorise les interactions fréquentes et étroites entre les collaborateurs (selon la courbe d'Allen, la communication perd son efficacité quand plus de 8 mètres séparent les interlocuteurs) ${ }^{2}$; 7) concentrer ses interventions sur les comportements exemplaires et les résultats remarquables; 8) mesurer les indicateurs qui comptent vraiment; 9) définir, hiérarchiser et communiquer clairement les priorités du groupe; 10) favoriser une culture et mettre au point un mécanisme qui assurent le « kaizen » (l'amélioration et le changement en continu); 11) garder à l'esprit que la satisfaction est plus importante que le profit (effet Hawthorne : pour la plupart des gens, la récompense émotionnelle importe plus que la récompense matérielle) ${ }^{3} ; 12$ ) faire preuve d'intégrité (montrer l'exemple et respecter ses engagements); et 13) préparer son organisation ou son équipe à réagir intelligemment en cas de situation imprévue, pour qu'elle puisse faire face à l'incertitude malgré les tâches qu'elle avait à accomplir ${ }^{3}$. Il est aussi important de rappeler que la diversité est essentielle non seulement pour une question d'égalité, mais aussi pour renforcer la capacité de l'équipe à résoudre des problèmes complexes et à maximiser les résultats. Scott Page a superbement décrit le phénomène dans son théorème de la diversitét. Enfin, il y a lieu de faire une distinction entre leader et gestionnaire. Dans le milieu de la santé, on les confond souvent. Les leaders sont rares; les gestionnaires, légions.

Idéalement, en temps normal, tout leader du milieu chirurgical doit s'efforcer de suivre chacun de ces principes. Mais qu'advient-il lorsque survient un événement inattendu comme une pandémie? En quoi cela vient-il bousculer les concepts traditionnels du leadership? Comme l'ont répété de nombreux boxeurs champions du monde, « on a tous un plan jusqu'à ce qu'une tuile nous tombe dessus ». En contexte pandémique, un leadership efficace doit prendre une autre forme. Carlos Pellegrini, un ancien président de l'American College of Surgeons, a dit récemment qu'il s'agit « non pas d'un leadership normal, mais d'un leadership de crise ${ }^{5} \gg$. Le $\mathrm{D}^{\mathrm{r}}$ Pellegrini a aussi savamment formulé une série de nouveaux principes : 1) être présent (être visible est la meilleure chose à faire pour apaiser l'anxiété et l'incertitude en temps de crise); 2) communiquer fréquemment avec ses collaborateurs (faire souvent des mises à jour et expliquer les mesures à prendre en conséquence); 3) transmettre toute l'information (en donner plus que moins, et entrer en contact avec l'équipe plutôt que de lui donner des ordres); 4) dire la vérité (le personnel se renseigne beaucoup de son côté, donc il vaut mieux opter pour la surinformation et l'honnêteté); et 5) afficher un optimisme pragmatique (la vérité ancrée dans la réalité $)^{5}$. L'efficacité de la gestion de crise passe par l'application de ces principes, auxquels on peut ajouter une dose de résilience et de patience.

Pour dire vrai, la pandémie de COVID-19 a certes eu des répercussions négatives, mais elle représente aussi une occasion. Comme l'a souligné le coach Woodward, « le succès repose sur la capacité d'une équipe à travailler 
collectivement sous pression, sur sa compréhension de l'importance de l'esprit d'équipe et sur sa volonté de toujours améliorer les choses, ne serait-ce qu'un tout petit $\mathrm{peu}^{6} \gg$. Une période de crise et de stress peut aussi améliorer les résultats d'une équipe grâce à un leadership exceptionnel ${ }^{7}$. Le ciment émotionnel d'un groupe ou d'une culture demeure le fait d'avoir une identité ou une mission communes, ou, dans le cas de la COVID-19, un ennemi commun. Au bout du compte, « les grands leaders protègent farouchement les leurs, favorisent les échanges et la collaboration et nourrissent le sentiment d'appartenance. Ils arrivent à créer un climat de confiance et de respect, comme au sein d'une famille ${ }^{1} \gg$. Pour reprendre les mots de Charles Darwin, « ce ne sont pas les espèces les plus fortes ou les plus intelligentes qui survivent, mais plutôt celles qui s'adaptent le mieux au changement $\gg$. Il nous faut assurer le rapprochement, la collaboration et la communication au sein de nos équipes chirurgicales pendant la présente crise, pour qu'elle fasse de nous des professionnels de la santé plus vigilants, plus efficaces et plus résilients.

\section{Chad G. Ball, MD, MSc}

Corédacteur en chef, fournal canadien de chirurgie

Intérêts concurrents : None declared.

DOI: $10.1503 /$ cjs. 016120

\section{Références}

1. Kerr J. Legacy. Constable \& Robinson LTD; 2015.

2. Allen TJ. Managing the flow of technology: technology transfer and the dissemination of technological information within the R\&D organization. Cambridge: MA MIT Press; 1984.

3. Coyle D. The culture code: The secrets of highly successful groups. Penguin Random House; 2017.

4. Page S. The difference: how the power of diversity creates better groups, firms, schools and societies. Princeton, NJ: Princeton University Press; 2007.

5. Hoyt DB. Looking forward. Bulletin of the fournal of the American College of Surgeons. 2020. Disponible : https://bulletin.facs.org/2020/07/ looking-forward-july-2020/ (consulté le 10 août 2020).

6. Woodward C. Winning! Hodder; 2005.

7. Walsh B. The score takes care of itself. Portfolio/Penguin; 2010. 